Old Dominion University ODU Digital Commons

Electrical \& Computer Engineering Faculty

Publications

Electrical \& Computer Engineering

2011

\title{
Anisotropic Response of Nanosized Bismuth Films Upon Femtosecond Laser Excitation Monitored by Uttrafast Electron Diffraction
}

\author{
Ahmed R. Esmail \\ Old Dominion University \\ Hani E. Elsayed-Ali \\ Old Dominion University, helsayed@odu.edu
}

Follow this and additional works at: https://digitalcommons.odu.edu/ece_fac_pubs

Part of the Nuclear Commons

\section{Repository Citation}

Esmail, Ahmed R. and Elsayed-Ali, Hani E., "Anisotropic Response of Nanosized Bismuth Films Upon Femtosecond Laser Excitation Monitored by Ultrafast Electron Diffraction" (2011). Electrical \& Computer Engineering Faculty Publications. 94.

https://digitalcommons.odu.edu/ece_fac_pubs/94

\section{Original Publication Citation}

Esmail, A. R., \& Elsayed-Ali, H. E. (2011). Anisotropic response of nanosized bismuth films upon femtosecond laser excitation monitored by ultrafast electron diffraction. Applied Physics Letters, 99(16), 161905. doi:10.1063/1.3652919 


\title{
Anisotropic response of nanosized bismuth films upon femtosecond laser excitation monitored by ultrafast electron diffraction
}

\author{
Ahmed R. Esmail ${ }^{\mathrm{a})}$ and Hani E. Elsayed-Ali ${ }^{\mathrm{b})}$ \\ Department of Electrical and Computer Engineering and the Applied Research Center, \\ Old Dominion University, Norfolk, Virginia 23529, USA
}

(Received 13 August 2011; accepted 21 September 2011; published online 18 October 2011)

\begin{abstract}
The lattice response of $5 \mathrm{~nm}$ thick bismuth film to femtosecond laser excitation is probed by ultrafast electron diffraction. The transient decay time after laser excitation is greater for diffraction from (012) lattice planes compared to (110) planes and is reduced for both planes with the increased laser fluence. These results indicate that different energy coupling mechanisms to the lattice occur depending on the crystal direction. The behavior of the diffraction peak width indicates partial disorder of the film upon photoexcitation that increases together with the laser fluence. (C) 2011 American Institute of Physics. [doi:10.1063/1.3652919]
\end{abstract}

Bismuth (Bi) crystallizes in the $\alpha$-arsenic structure which can be derived from the face-centered cubic structure with a weak rhombohedral distortion. ${ }^{1}$ That unit cell has two atoms: the first is on a lattice site, while the second is slightly displaced from the center along the body diagonal and is stabilized by the Peierls mechanism, which introduces a narrow band gap and renders $\mathrm{Bi}$ as a semimetal. ${ }^{2}$ If $\mathrm{Bi}$ is excited by an ultrafast laser pulse, the equilibrium distance of the two basis atoms is modified and the $\mathrm{Bi}$ atoms oscillate along the body diagonal generating the $\mathrm{A}_{1 \mathrm{~g}}$ coherent optical phonons. ${ }^{3}$ Bi has a very small electron effective mass tensor components, large mean-free path $(\sim 100 \mathrm{~nm}$ at $300 \mathrm{~K})$, highly anisotropic Fermi surface, and small energy overlap of $36 \mathrm{meV}$, making bulk $\mathrm{Bi}$ a semimetal. ${ }^{4}$ When the electrons are confined, the band energy split increases and results in the appearance of a small indirect band gap.,

We report here on the observation of anisotropy in lattice dynamics as detected in the electron diffraction intensity for the (012) and (110) diffraction peaks from a $5 \mathrm{~nm}$ thick Bi film. The transient decay time after laser excitation is greater for the (012) Bragg peak compared to the (110) peak. The behavior of the width of the Bragg peaks with time indicates partial disorder of the $\mathrm{Bi}$ flat islands upon photoexcitation.

Ultrafast electron diffraction (UED) ${ }^{6-8}$ was used to probe the structure of laser-excited $\mathrm{Bi}$ thin film. A $35-\mathrm{keV}$ electron gun was used to produce electron pulses by photoemission from a silver thin-film photocathode excited by frequency-tripled laser pulses (110 fs duration, $800 \mathrm{~nm}$ fundamental wavelength, at $1 \mathrm{kHz}$ repetition rate). The Bi film was exposed to the fundamental wavelength. The temporal resolution of the UED setup is better than 2 ps at the photocathode excitation level used. This was confirmed by performing UED experiment on $20 \mathrm{~nm}$ thick free-standing aluminum and bismuth films which showed lattice thermalization time in good agreement with that reported previously. ${ }^{9,10}$ The UED system is operated in low $10^{-9}$ Torr.

\footnotetext{
a) Physics Department, Cairo University, Cairo, Egypt.

b) Author to whom correspondence should be addressed. Electronic mail: helsayed@odu.edu. Telephone: (757)683-3748.
}

The Bi films were grown by thermal evaporation on $3 \mathrm{~mm}$ diameter copper transmission electron microscopy (TEM) grids coated with $<10 \mathrm{~nm}$ thick carbon layers. The as-deposited flat islands were annealed by femtosecond pulsed laser with a fluence of $2.4 \mathrm{~mJ} / \mathrm{cm}^{2}$. Laser annealing with a fluence of $3.3 \mathrm{~mJ} / \mathrm{cm}^{2}$ had similar annealing effect. The laser-annealed sample had a diffraction pattern that remained stable during the reported experiments and recovered after heating. Figure 1 shows TEM images of the asdeposited $\mathrm{Bi}$ film and after laser annealing, along with the corresponding transmission electron diffraction patterns. The lattice parameters remained unaffected by annealing and were found to be $a=4.6 \pm 0.1 \AA$ and $c=11.6 \pm 0.1 \AA$, which are in good agreement with the reported values of $4.54 \AA$ and $11.81 \AA$ for the Bi unit cell. ${ }^{11}$

Figure 2 shows the normalized diffraction intensity of the (012) and (110) Bragg peaks for two laser fluences,
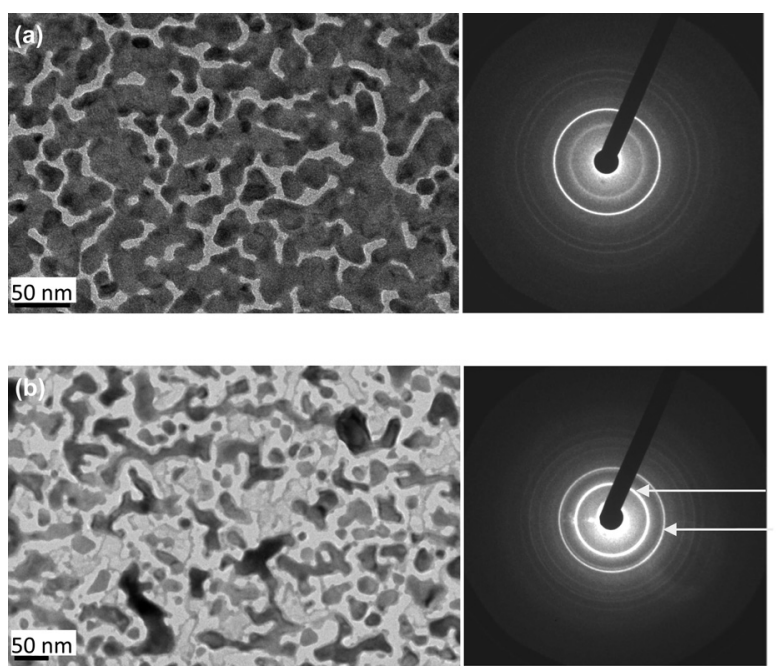

FIG. 1. HRTEM images and diffraction pattern of as-deposited $5 \mathrm{~nm}$ thick Bi film (a) and laser annealed sample at laser fluence of $2.4 \mathrm{~mJ} / \mathrm{cm}^{2}$ operated at $1 \mathrm{kHz}$ repetition rate (b). Laser annealing causes some agglomeration of the as-deposited islands and an increase of voids. The diffraction patterns are characteristic of the bulk hexagonal unit cell, indicating no structural phase transition occurring during annealing. The ring intensities change by annealing indicating changes in film orientation. 

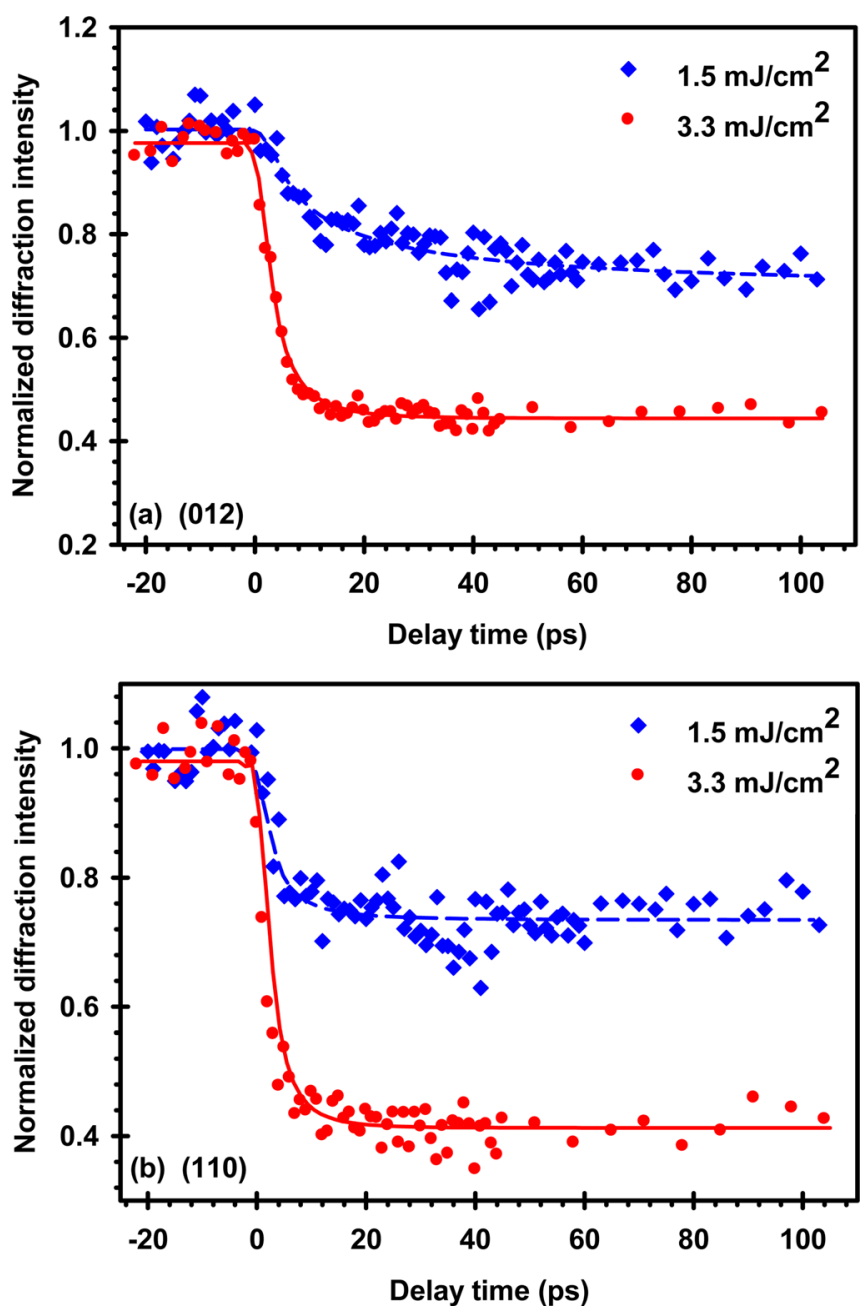

FIG. 2. (Color online) Time evolution of Bragg peak normalized intensity. (a) For the (012) planes, the decay time $\tau$ is 11.2 and $4.1 \mathrm{ps}$ for laser fluence of 1.5 and $3.3 \mathrm{~mJ} / \mathrm{cm}^{2}$, respectively. (b) For the (110) planes, $\tau$ is 4.3 and 3.1 ps for laser fluence of 1.5 and $3.3 \mathrm{~mJ} / \mathrm{cm}^{2}$, respectively. Anisotropy in the decay time of the (012) and (110) peaks indicates different lattice excitation mechanisms that is dependent on crystal direction.

1.5 and $3.3 \mathrm{~mJ} / \mathrm{cm}^{2}$. The decay time $\tau$ of the normalized intensity is found to be dependent on the laser fluence and the diffraction peak. It is generally assumed that the decay of the diffraction intensity in UED follows the Debye-Waller treatment where the intensity of diffraction peak is reduced due to increased single- and multi-phonon scattering by lattice heating. This is considered valid in analysis of UED experiments as a measure of the mean vibrational amplitude; $;^{14,15}$ however, extension of this treatment to define a temperature is not valid when the equilibrium phonon distribution has not yet been reached. Phonon equilibration can take several tens of picoseconds. UED measurements of the decay of coherent acoustic phonons in Bi film showed a decay time of 40-50 $\mathrm{ps},{ }^{12}$ which is four times longer than previously found for optical phonons in pump-probe reflectivity measurements. ${ }^{13}$ Therefore, the time required for the phonons to reach equilibrium in the $\mathrm{Bi}$ film is longer than $\sim 50 \mathrm{ps}$ for the experimental conditions used.

The decay time $\tau$ in Fig. 2 was fitted to a single exponential. Considerable anisotropy in $\tau$ is observed between the (012) and (110) Bragg peaks. At a laser fluence of
$1.5 \mathrm{~mJ} / \mathrm{cm}^{2}, \tau$ was $11.2 \mathrm{ps}$ for the (012) peak and $4.3 \mathrm{ps}$ for the (110) peak, while at $3.3 \mathrm{~mJ} / \mathrm{cm}^{2}, \tau$ was 4.1 and $3.1 \mathrm{ps}$ for the (012) and (110) peaks, respectively. This anisotropy in $\tau$ shows that the energy transfer from the excited carriers and coherent phonons to the lattice is anisotropic. Since the thermalization of the acoustic phonons takes much longer than $\tau$, the anisotropy in the energy transfer rate to the lattice will appear as a difference in $\tau$ measured for different planes. Lattice excitation occurs through carrier thermalization by electron-phonon and phonon-phonon interactions. Carrier diffusion is negligible since the film thickness is less than the laser skin depth in Bi which is $\sim 28 \mathrm{~nm}^{13}$

Excitation of $\mathrm{Bi}$ by ultrashort laser pulses changes the equilibrium atomic positions within the unit cell by displacive excitation of coherent phonons of the symmetric $\mathrm{A}_{1 \mathrm{~g}}$ mode. The photoexcited carriers generate nonequilibrium electron distribution that changes the lattice bonding. The main mechanism for the relaxation of the $\mathrm{A}_{1 \mathrm{~g}}$ coherent phonons is dephasing by phonon-phonon interaction due to lattice anharmonicity. ${ }^{16}$ The carrier relaxation time and dephasing time of the $\mathrm{A}_{\mathrm{gg}}$ phonons were previously found to depend on the structure and size of the studied sample. For a polycrystalline Bi film, these times were both $\sim 4.1 \mathrm{ps}$. The carrier relaxation time was shorter (2.8 ps) in Bi single crystal, while the $A_{1 g}$ phonons dephasing time was about the same as for the polycrystalline sample. ${ }^{16}$ The increased carrier relaxation time in the polycrystalline sample is thought to be due to its semiconductor character as a result of size reduction.

The decay time $\tau$ observed for the (110) peak is consistent with the carrier relaxation time observed in Bi films. ${ }^{16}$ The (110) planes are parallel to the body diagonal and, therefore, diffraction from these planes is not sensitive to $A_{1 g}$ oscillations or decay of these oscillations into coherent acoustic phonons oscillating along the body diagonal. However, the (012) peak is sensitive to oscillations along the body diagonal. The longer $\tau$ observed for the (012) peak could be due to additional lattice excitation mechanisms involving decay of $\mathrm{A}_{1 \mathrm{~g}}$ oscillations into coherent acoustic phonons and their subsequent relaxation into the phonon bath.

Increasing the laser fluence from 1.5 to $3.3 \mathrm{~mJ} / \mathrm{cm}^{2}$ reduced $\tau$ for the observed diffraction peaks. The reduction of $\tau$ together with increasing fluence was also previously observed for $30 \mathrm{~nm}$ thick Bi film, although no anisotropy in $\tau$ was reported for the diffraction peaks studied. ${ }^{10}$ The reduction in $\tau$ together with increasing laser fluence can be explained by the increased carrier density resulting in higher carrier relaxation time, as well as by an increase in the damping of the coherent phonons, both optical and acoustic, with laser fluence as was previously detected for the $\mathrm{A}_{1 \mathrm{~g}}$ oscillations. ${ }^{17}$

To estimate the final lattice temperature, we used the normalized diffraction intensity level near the end of the pump-probe scans in Fig. 2 and the measured Debye temperature for the (110) diffraction peaks, which we found previously to be $86 \pm 9 \mathrm{~K}^{18}$ The increase in lattice temperature as a result of heating by $1.5 \mathrm{~mJ} / \mathrm{cm}^{2}$ laser pulse is $\sim 150 \mathrm{~K}$, resulting in maximum temperature of $\sim 450 \mathrm{~K}$. Surface melting of the $\mathrm{Bi}$ islands can occur at this temperature. ${ }^{18,19}$ 


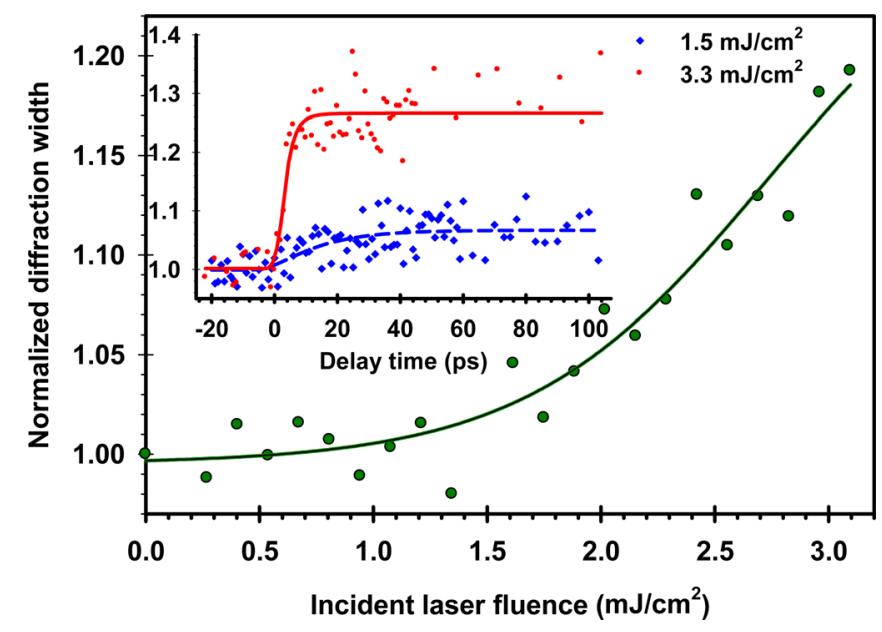

FIG. 3. (Color online) Diffraction width of the (012) Bragg peak normalized to that without laser heating taken at delay time 41 ps for different laser fluences. Inset is time evolution of normalized Bragg peak width at two different laser fluences.

Figure 3 shows the full width at half maximum (FWHM) of the (012) diffraction peak at the time delay of 41 ps normalized to that without laser heating plotted as a function of the laser fluence. The behavior of the (110) peak was similar in shape. The increase in the FWHM of the diffraction peak width indicates reduction in the crystalline size. We have previously observed an increased diffraction peak width in the case of Bi nanoparticles under continuous heating, and this was attributed to formation of a liquid shell around the nanoparticles. ${ }^{18}$ Excitation of longitudinal acoustic phonons and strain effects can also affect the measured FWHM, however, unlike the behavior observed in Fig. 3, these effects decay in a few tens of picosecond. ${ }^{20}$ The results in Fig. 3 show that some disorder is occurring at a fluence as low as $1.5 \mathrm{~mJ} / \mathrm{cm}^{2}$ and continues to increase with the laser fluence. The inset in Fig. 3 shows the temporal development of the normalized FWHM, which shows that the diffraction width reaches a plateau at a time that is reduced with the increasing laser fluence and is delayed by $\sim 20 \mathrm{ps}$ for the laser fluence of $1.5 \mathrm{~mJ} / \mathrm{cm}^{2}$. The FWHM remains constant up to the maximum probed time, $100 \mathrm{ps}$. This observation is consistent with the formation of a stable disorder (a liquid layer), that persists over time due to the slow thermal diffusion out of the Bi islands.

In UED experiments on $5 \mathrm{~nm}$ thick Bi film, consisting of flat islands, the temporal development of the diffraction intensity shows that the transient decay time after laser excita- tion is greater for the (012) diffraction peak compared to the (110) peak. These results indicate that different energy coupling mechanisms, depending on the crystal direction, are occurring in the case of femtosecond laser excitation. The behavior of the FWHM of the diffraction peaks with time indicates partial disorder of the $\mathrm{Bi}$ islands that increases with the laser fluence.

This material is based upon work supported by the U.S. Department of Energy Grant No. DE-FG02-97ER45625 and National Science Foundation Grant Nos. DMR-9988669 and MRI-0821180.

${ }^{1}$ Landolt-Börnstein, New Series, Group III: Crystal and Solid State Physics, Semiconductors Vol. 17a, edited by O. Madelung (Springer, Berlin, 1983).

${ }^{2}$ R. E. Peierls, More Surprises in Theoretical Physics (Princeton University, Princeton, 1991), pp. 24-26.

${ }^{3}$ H. J. Zeiger, J. Vidal, T. K. Cheng, E. P. Ippen, G. Dresselhaus, and M. S. Dresselhaus, Phys. Rev. B 45, 768 (1992).

${ }^{4}$ M. R. Black, P. L. Hagelstein, S. B. Cronin, Y. M. Lin, and M. S. Dresselhaus, Phys. Rev. B 68, 235417 (2003).

${ }^{5}$ M. R. Black, M. Padi, S. B. Cronin, Y.-M. Lin, O. Rabin, T. McClure, G. Dresselhaus, P. L. Hagelstein, and M. S. Dresselhaus, Appl. Phys. Lett. 77, 4142 (2000).

${ }^{6}$ H. E. Elsayed-Ali and J. W. Herman, Rev. Sci. Instrum. 61, 1636 (1990).

${ }^{7}$ H. E. Elsayed-Ali, 23rd International Congress on High-Speed Photography and Photonics, SPIE Proceedings Vol. 3516, edited by V. P. Degtyareva, M. A. Monastyrski, M. Y. Schelev, and A. V. Smirnov, (P. N. Lebedev Physical Institute, Moscow, Russia, 1998), pp. 607-615.

${ }^{8}$ B.-L. Qian and H. E. Elsayed-Ali, J. Appl. Phys. 91, 462 (2002).

${ }^{9}$ H. Park, X. Wang, S. Nie, R. Clinite, and J. Cao, Solid State Commun. 136, 559 (2005).

${ }^{10}$ G. Sciaini, M. Harb, S. Kruglik, T. Payer, C. Hebeisen, F. Heringdorf, M. Yamaguchi, M. Horn-von Hoegen, R. Ernstorfer, and R. J. Dwayne Miller, Nature 458, 56 (2009).

${ }^{11}$ X. F. Yu, X. Liu, K. Zhang, and Z. Q. Hu, J. Phys.: Condens. Matter 11, 937 (1999).

${ }^{12}$ A. Bugayev, A. Esmail, M. Abdel-Fattah, and H. E. Elsayed-Ali, AIP Advances 1, 012117 (2011).

${ }^{13}$ D. Boschetto, E. G. Gamaly, A. V. Rode, B. Luther-Davies, D. Glijer, T. Garl, O. Albert, A. Rousse, and J. Etchepare, Phys. Rev. Lett. 100, 027404 (2008).

${ }^{14}$ H. E. Elsayed-Ali, U.S. patent 5,010,250 (23 April 23 1991).

${ }^{15}$ H. E. Elsayed-Ali and J. W. Herman, Appl. Phys. Lett. 57, 1508 (1990).

${ }^{16}$ H. Hase, K. Ishioka, M. Kitajima, S. Hishita, and K. Ushida, Appl. Surf. Sci. 197-198, 710 (2002).

${ }^{17}$ A. A. Mel'nikov, O. V. Misochko, and S. V. Chekalin, JETP Lett. 89, 129 (2009).

${ }^{18}$ A. Esmail, M. Abdel-Fattah, and H. E. Elsayed-Ali, J. Appl. Phys. 109, 084317 (2011).

${ }^{19}$ M. K. Zayed and H. E. Elsayed-Ali, J. Appl. Phys. 99, 123516 (2006).

${ }^{20} \mathrm{M}$. Bargheer, N. Zhavoronkov, M. Woerner, and T. Elsaesser, ChemPhys Chem 7(4), 783 (2006). 